\title{
OPEN Transpapillary tissue sampling of biliary strictures: balloon dilatation prior to forceps biopsy improves sensitivity and accuracy
}

\author{
Daniel Pörner ${ }^{1}$, Dominik J. Kaczmarek ${ }^{1}$, Dominik Heling ${ }^{1}$, Annekristin Hausen ${ }^{1}$, \\ Raphael Mohr ${ }^{1}$, Robert Hüneburg ${ }^{1}$, Hanno Matthaei ${ }^{2}$, Tim R. Glowka², Steffen Manekeller ${ }^{2}$, \\ Hans-Peter Fischer ${ }^{3}$, Marieta Toma ${ }^{3}$, Jacob Nattermann ${ }^{1}$, Christian P. Strassburg ${ }^{1}$, \\ Maria A. Gonzalez-Carmona ${ }^{1} \&$ Tobias J. Weismüller ${ }^{1 \bowtie}$
}

The early and definitive diagnosis of malignant bile duct stenoses is essential for a timely and adequate therapy. However, tissue sampling with transpapillary brush cytology (BC) or forceps biopsy (FB) remains challenging. With this study, we aimed to compare the effectiveness and safety of different tissue sampling modalities (BC, FB without/after previous balloon dilatation). Standardized database research identified all patients, who underwent endoscopic retrograde cholangiography with BC and/or FB for indeterminate bile duct stenosis between January 2010 and April 2018 and with a definitive diagnosis. 218 patients were enrolled (149 cases with malignant and 69 with benign disease). FB had a significant higher sensitivity than BC ( $43 \%$ vs. $16 \%, \mathrm{p}<0.01)$. Prior balloon dilatation of the stenosis improved the sensitivity of FB from 41 to $71 \%(p=0.03)$, the NPV from 36 to $81 \%$ $(p<0.01)$ and the accuracy from 55 to $87 \%(p<0.01)$. The complication rates did not differ significantly between the modalities. In our center FB turned out to be the diagnostically more effective procedure. Balloon dilatation of the stenosis before FB had a significant diagnostic benefit and was not associated with a higher complication rate.

Biliary strictures are frequently caused by malignant tumors of the intra- or extrahepatic biliary tree, the gallbladder, the liver, the pancreas or the surrounding lymph nodes or by metastasis in these organs. However, benign conditions like autoimmune or infectious diseases or posttraumatic ischemias can also induce inflammation and scarring fibrosis, that mimic a malignant stricture. Unless the underlying history and the typical localization and configuration of the stricture unambiguously indicate a benign etiology, we speak of an indeterminate biliary stricture (IBiS), which is highly suspicious for malignancy but lacks a confirmative tissue diagnosis ${ }^{1}$. This is often the case in patients with primary sclerosing cholangitis (PSC), who develop fibrotic bile duct strictures but have a risk of $1.4 \%$ per year ${ }^{2}$ and a life-time risk of up to $14 \%^{3}$ to develop hepatobiliary malignancies, and in patients with chronic pancreatitis, who frequently develop strictures due to inflammation of the pancreatic head but also have an increased risk of pancreatic cancer (PCA $)^{4}$.

The early and definitive exclusion or securing of malignancy of bile duct strictures is essential in order to avoid overtreatment (e.g. non-indicated surgical interventions or non-indicated chemotherapies) on the one hand, but on the other hand to allow for a curative resection or at least an immediate start of a palliative chemotherapy.

Current guidelines ${ }^{5,6}$ emphasize the importance of additional imaging like endoscopic ultrasound (EUS), computed tomography (CT) or magnetic resonance imaging (MRI) for the characterization and, if necessary and possible, targeted biopsy of a stricture-related mass. Furthermore, a potential benefit of additional endoscopic tools like cholangioscopy, intraductal ultrasound or laser endomicroscopy is discussed. Despite these methods, however, it is still the primary goal to obtain enough tissue for histological or cytological diagnosis. Since endoscopic retrograde cholangiography (ERC) is indicated under most circumstances, transductal brush cytology (BC) and transpapillary forceps biopsy (FB) are the methods of choice for tissue sampling but both with limited

${ }^{1}$ Department of Internal Medicine I, University Hospital Bonn, Venusberg-Campus 1, 53127 Bonn, Germany. '2Department of Surgery, University Hospital Bonn, Venusberg-Campus 1, 53127 Bonn, Germany. ${ }^{3}$ Institute of Pathology, University Hospital Bonn, Venusberg-Campus 1, 53127 Bonn, Germany. ${ }^{\circledR}$ email: Tobias.Weismueller@ukbonn.de 
endoscopic database research with the following criteria:

1) suspected malignant biliary stricture

2) brush cytology and/or forceps biopsy

3) in ERCs from January 2010 to April 2018

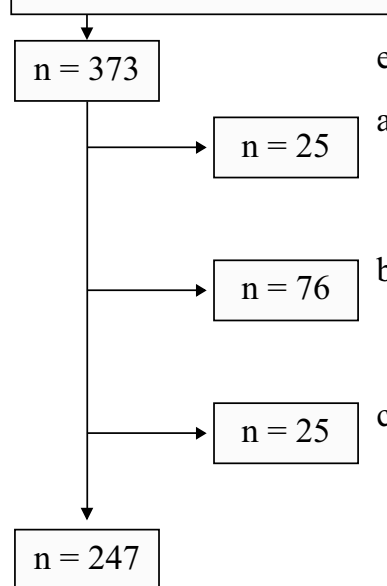

exclusion due to:

a) suspicious stricture was diagnosed before

January 2010

b) no $\mathrm{BC} / \mathrm{FB}$ conducted:

other diagnostic

modality preferred

c) no $\mathrm{BC} / \mathrm{FB}$ conducted:

declined by patient,

palliative situation,

other reasons

218 patients with verified dignity

(stricture dignity could not be verified in 29 cases)

Figure 1. Patient enrollment. $E R C$ endoscopic retrograde cholangiography, $B C$ brush cytology, $F B$ forceps biopsy.

overall sensitivity. In the present study we aimed to identify the best diagnostic strategy considering relevant co-variates (type of forceps, localization of stricture, previous intervention) and complications.

\section{Methods}

Through a structured database query of our endoscopy documentation system (Viewpoint 5, GE Healthcare) we identified all patients, who were first diagnosed with a biliary stricture suspicious for malignancy between January 2010 and April 2018 and underwent ERC with BC and/or FB at the University Hospital Bonn (Fig. 1). Strictures were considered suspicious for malignancy based on patient's history and symptoms (e.g. painless jaundice, history of PSC, no previous surgery/trauma) and/or cholangiographic features including an irregular margin, asymmetric or abrupt narrowing and the double-duct sign (see Fig. 2A).

Choice and order of the diagnostic tools ( $\mathrm{BC}$ and/or $\mathrm{FB}$ ) depended on the assessment and preference of the respective endoscopist. For BC we used a 6 Fr cytology brush (Cook Medical Fusion Cytology Brush, length $25 \mathrm{~mm}$, Fig. 2B). For FB, two different forceps types were used: Larger forcepses (Endo-Flex Biopsy Forceps, outer diameter $2.3 \mathrm{~mm}$, oval cups and spike) were preferably used for strictures of the common bile duct (CBD) or the hilar region and were positioned free-handed under fluoroscopic control (Fig. 2F). For strictures of the intrahepatic ducts, usually smaller forcepses (MTW Biopsy Forceps, outer diameter $1.8 \mathrm{~mm}$, oval spoon-shaped mouth without spike) were advanced through a $10 \mathrm{Fr}$ pushing catheter (Endo-Flex Pusher, Fig. 2C) as described previously ${ }^{7}$. Strictures were frequently dilated with a balloon (Cook Medical Fusion Titan Biliary Dilation Balloon, 4-10 mm) before or after taking 1-3 biopsies (Fig. 2D-F). In most cases, biliary stents (Boston Scientific Flexima endoprostheses, 7-11.5 Fr; Endo-Flex double-pigtail stents, 7 or $10 \mathrm{Fr}$ ) were placed to secure bile flow.

We retrospectively recorded patient characteristics (i.e. age, gender, pre-existing diseases, prior surgery involving the hepatobiliary tract, previously diagnosed malignoma), ERC procedure details (i.e. modalities for tissue sampling including chronological order, previous dilatation, sphincterotomy or stenting, duration of ERC), stricture characteristics (localization, length, extent), ERC-induced complications (pancreatitis, cholangitis, hemorrhage with requirement of blood transfusions, perforation) and pre-interventional CA 19-9 levels.

Final diagnosis of malignancy was based on (a) cytologic and/or histologic evidence obtained by tissue sampling during ERC, endoscopic or percutaneous fine needle biopsy (FNB), surgery or autopsy; or (b) clinical course during a follow-up of at least six months. Definitely malignant and severely suspicious cyto-/histopathological findings were classified as positive. Nearly all tissue samples acquired via $\mathrm{BC}$ or FB were examined by two of our local pathologists with appropriate expertise. In cases when $\mathrm{BC}$ and $\mathrm{FB}$ were conducted during the same ERC, tissue samples from both modalities were examined by the same pathologists. A stricture was considered benign when there was no evidence for malignancy during a follow-up of at least 6 months (i.e. absence of radiomorphologic tumor progression including infiltration and/or metastatic dissemination). Unless otherwise specified, the following analyses are based on each patient's first ERC in domo that fulfills the above mentioned inclusion criteria. The study was performed in accordance with the Declaration of Helsinki (as revised in 2013) and was approved by the Ethics Committee of the Medical Faculty of the University of Bonn (approval number 

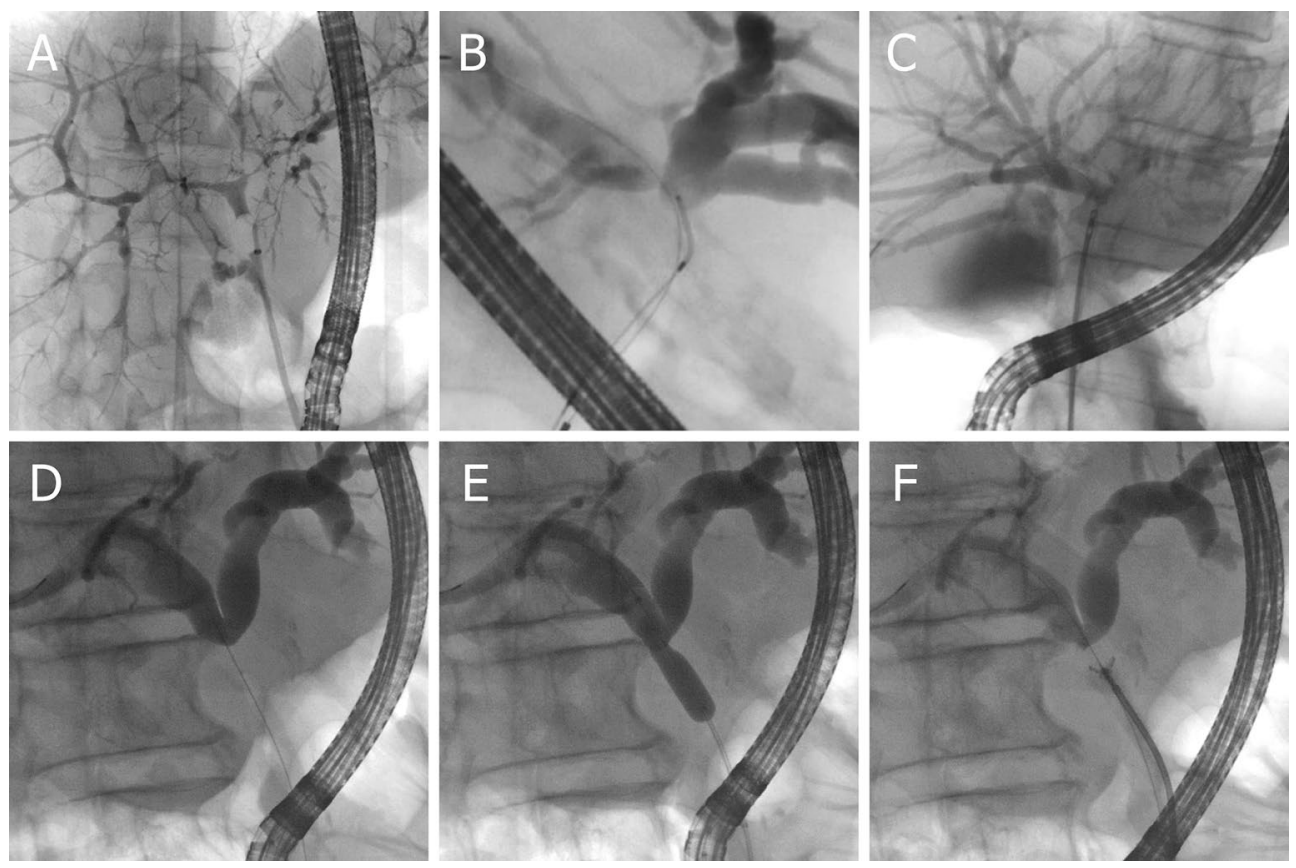

Figure 2. Biliary strictures and different modalities for tissue sampling. (A) Dominant stricture of the left intrahepatic duct in a patient with advanced primary sclerosing cholangitis. (B) Acquisition of a brush cytology of a hilar stricture. (C) Small diameter forceps biopsy of a hilar stricture. (D-F) Cholangiography revealed a highly suspicious stricture of the proximal common bile duct in a patient undergoing endoscopic retrograde cholangiography due to obstructive jaundice. After a guide-wire had been passed over the stricture (D), balloon dilatation of the stricture was performed (E). Subsequently, multiple specimens were obtained using a large diameter forceps $(\mathbf{F})$. Histopathologic examination exposed the stricture to be caused by a cholangiocarcinoma.

233/17). Written informed consent for ERC including tissue sampling and stricture dilatation was obtained from all patients.

Statistical analysis. Categorical variables are expressed as numbers with percentages in parenthesis. Continuous data are presented as mean \pm standard deviation. Statistical comparison of proportions was performed using $\mathrm{Chi}^{2}$ test (with Yates correction) and two-tailed Fisher's exact test, respectively. Unpaired $t$ test with Welch's correction was used to compare means of continuous variables. A p-value less than 0.05 was considered statistically significant. Statistical analyses were performed with IBM SPSS 25.

\section{Results}

Patients' characteristics and final diagnosis. Endoscopic database research yielded a total of 247 patients with IBiS, who underwent ERC from January 2010 to April 2018 and received further sampling with BC and/or FB (Fig. 1). No definitive diagnosis could be obtained in 29 cases due to an insufficient follow-up, i.e. less than 6 months (for death or other reasons). Therefore, 218 patients (Table 1) remained for the final statistical analysis.

Malignant disease was finally diagnosed in 149 patients. Final malignant diagnosis based on (a) cytological and/or histological evidence obtained by surgery/autopsy $(n=81)$, percutaneous tissue sampling $(n=18)$, EUS-FNA $(n=2)$, tissue sampling via BC/FB $(n=38)$ and (b) the clinical course during a follow-up of 6 months ( $n=10$, mean follow-up for this cohort: $12.59 \pm 7.10$ months). Cases with confirmation of malignancy by multiple diagnostic modalities were subsumed under the first mentioned method. Benign condition was finally diagnosed in 69 patients by (a) surgery $(n=24)$ and (b) the clinical course during a follow-up of at least 6 months $(n=45$, mean follow-up for this cohort: $27.15 \pm 18.10$ months).

The underlying diseases are summarized in Table 2. Malign stricture was caused by cholangiocarcinoma (CCA) in 89 cases (59.73\%) and by PCA in 38 cases (25.50\%). PSC was the most frequent cause of benign stricture with $50.72 \%$ (35/69 cases). $40 / 218$ patients (18.35\%) fulfilled inclusion criteria for the study due to PSC with dominant stricture (Fig. 2A). In 5 of these cases (12.50\%), dominant stricture was caused by a cholangiocarcinoma. $70 / 218$ patients $(32.11 \%), 44$ patients with benign and 26 patients with malignant stricture, underwent more than one ERC during the evaluation period (Fig. 3).

Overall diagnostic performance of BC and FB. Altogether 83 brush cytologies and 188 forceps biopsies were obtained during the first ERC with tissue sampling in 218 patients. Sufficient sample material for pathological examination was acquired in $85.54 \%$ (71/83 BC) and 97.87\% (184/188 FB). Definitely malignant or highly suspicious pathological findings were acquired with BC in 7 of 44 (15.91\%) and with FB in 56 of 129 


\begin{tabular}{|l|l|l|l|}
\hline & Benign stricture $(\mathbf{n}=\mathbf{6 9})$ & Malignant stricture $(\mathbf{n}=\mathbf{1 4 9})$ & All strictures $(\mathbf{n}=\mathbf{2 1 8})$ \\
\hline Age (years) & $52.50^{*} \pm 17.26$ & $68.54^{*} \pm 12.02$ & $63.46 \pm 15.74$ \\
\hline Sex (female/male) & $26 / 43$ & $60 / 89$ & $86 / 132$ \\
\hline Mean follow-up (months) & $28.52^{\star} \pm 21.16$ & $13.30^{*} \pm 14.30$ & $18.12 \pm 18.17$ \\
\hline Pre-interventional CA 19-9 (U/ml) & $92.95^{\star} \pm 365.98$ & $4218.72^{\star} \pm 11,828.27$ & $3081.70^{*} \pm 10,222.82$ \\
\hline Pre-existing conditions/prior surgery of the hepatobiliary tract & \multicolumn{2}{|l|}{} \\
\hline PSC & $35 / 69^{*}$ & $5 / 149^{*}$ & $40 / 218$ \\
\hline SSC/IAC & $6 / 69^{*}$ & $2 / 149^{*}$ & $8 / 218$ \\
\hline Chronic pancreatitis & $6 / 69$ & $4 / 149$ & $10 / 218$ \\
\hline Choledocholithiasis & $20 / 69^{*}$ & $17 / 149^{*}$ & $37 / 218$ \\
\hline Prior cholecystectomy & $17 / 69$ & $37 / 149$ & $54 / 218$ \\
\hline Cholecystolithiasis & $10 / 49^{\dagger}$ & $28 / 107^{\dagger}$ & $38 / 156^{\dagger}$ \\
\hline Prior partial hepatectomy & $2 / 69$ & $2 / 149$ & $4 / 218$ \\
\hline Previously diagnosed extrahepatobiliary malignoma & $14 / 69$ & $42 / 149$ & $56 / 218$ \\
\hline
\end{tabular}

Table 1. Patients' characteristics and pre-existing conditions. PSC primary sclerosing cholangitis, SSC secondary sclerosing cholangitis, IAC IgG4-associated cholangitis. ${ }^{*} \mathrm{p}<0.05$ for comparison benign vs. malignant strictures, ${ }^{\dagger}$ presence of cholecystolithiasis unknown in 3 cases with benign stricture and 5 cases with malignant stricture.

\begin{tabular}{|l|l|l|}
\hline & n & $\%$ \\
\hline Benign stricture & 69 & 100 \\
\hline PSC related benign stricture & 35 & 50.72 \\
\hline SSC related benign stricture & 4 & 5.80 \\
\hline IAC related benign stricture & 2 & 2.90 \\
\hline Chronic pancreatitis with benign stricture of the CBD & 3 & 4.35 \\
\hline Recurrent choledocholithiasis with benign stricture & 10 & 14.49 \\
\hline Postoperative benign stricture & 1 & 1.45 \\
\hline Others & 8 & 11.59 \\
\hline Unknown & 6 & 8.70 \\
\hline Malignant stricture & 149 & 100 \\
\hline Cholangiocarcinoma & 89 & 59.73 \\
\hline Pancreatic cancer & 38 & 25.50 \\
\hline Gallbladder cancer & 5 & 3.36 \\
\hline Hepatocellular carcinoma & 3 & 2.01 \\
\hline Metastasis of extrahepatobiliary primarius & 9 & 6.04 \\
\hline Others & 4 & 2.68 \\
\hline Unknown & 1 & 0.67 \\
\hline
\end{tabular}

Table 2. Final diagnosis. PSC primary sclerosing cholangitis, SSC secondary sclerosing cholangitis, IAC IgG4associated cholangitis, $C B D$ common bile duct.

(43.41\%) patients with malignant disease ( $\mathrm{p}<0.01$, Fig. 4). Corresponding negative predictive values (NPV) and accuracies were $42.19 \%$ and $47.89 \%$ for BC and $42.97 \%(p=0.92)$ and $60.33 \%(p=0.07)$ for FB. Specificity and positive predictive values reached $100 \%$ for both modalities (no false-positive diagnoses). BC and FB showed higher sensitivities for CCA compared to PCA (CCA: BC: 23.08\%, FB: 51.90\%, PCA: BC: 10\%, FB: 32.35\%, CCA vs. PCA: $B C: p=0.65, F B: p=0.06)$. The localization of CCA had no significant influence on the sensitivity of FB (distal CCA: 60\%, perihilar CCA: $46.15 \%$, intrahepatic CCA: $71.43 \%, p>0.05$ ). Due to a low case count in the subgroups, analyses of the sensitivity of BC depending on localization of CCA were not reliable.

Influence of stricture dilatation on the diagnostic performance of forceps biopsy. Balloon dilatation of the stricture was performed in $49 / 218$ cases (22.48\%). FB was performed after prior balloon dilatation in 31 cases and without prior dilatation in 158 cases (Fig. 4). Stricture dilatation to $6-10 \mathrm{~mm}$ (depending on the diameter of the bile duct) prior to FB provided a significant diagnostic advantage: Without prior dilatation, FB yielded a sensitivity of $40.52 \%$, an NPV of $35.51 \%$ and an accuracy of $55.19 \%$ (Fig. 4). After stricture dilatation, sensitivity, NPV and accuracy significantly increased to $71.43 \%(p=0.03), 80.95 \%(p<0.01)$ and $87.10 \%$ $(\mathrm{p}<0.01)$. In none of the analyzed procedures, balloon dilatation of the stricture was performed prior to BC. 


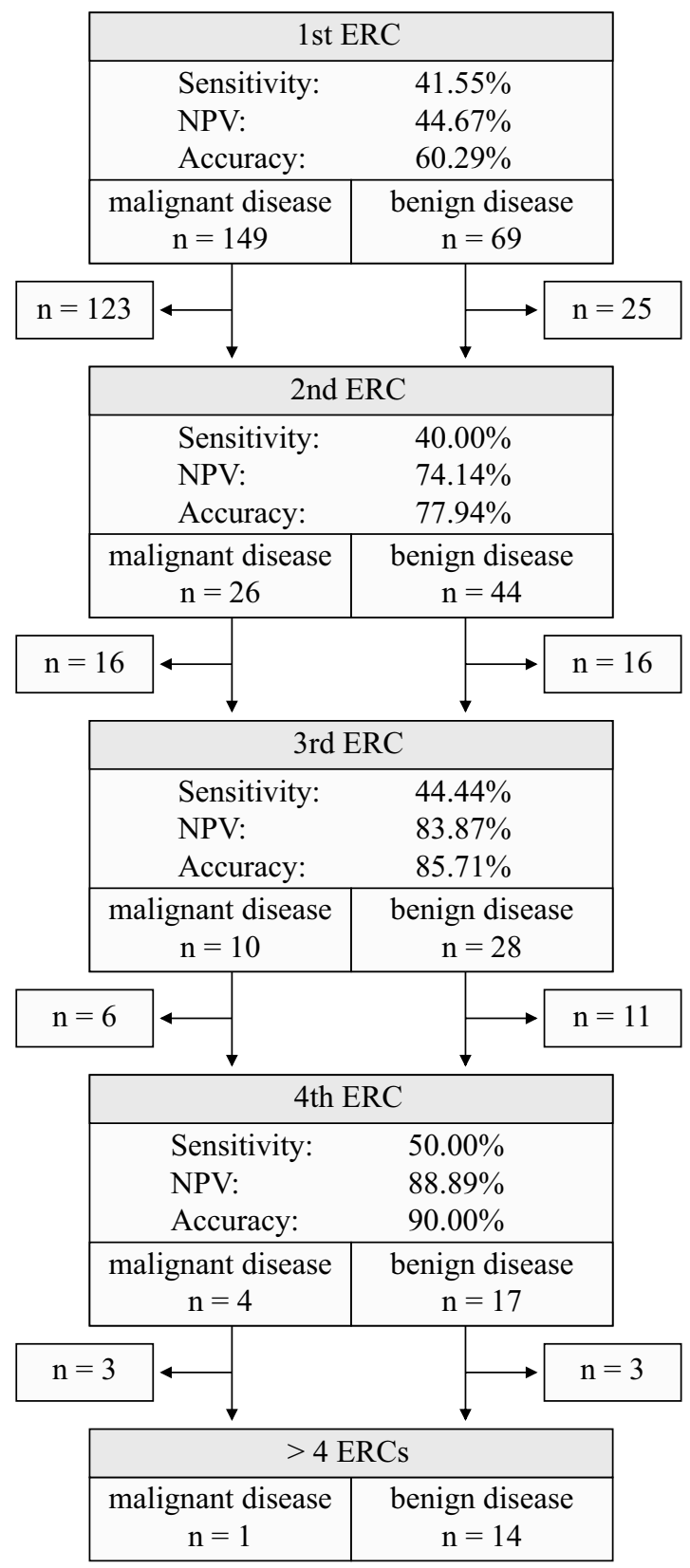

Figure 3. Follow-up ERCs. 70 patients received more than one endoscopic retrograde cholangiography (ERC). Patient drop out (as noted on the sides) was due to the clinical course of malignant disease, intercurrent confirmation of stricture dignity through other modalities or a loss of follow-up. There was only one patient with malignant disease among the 15 patients undergoing more than four ERCs. The denoted sensitivities, negative predictive values (NPV) and accuracies of the ERCs were calculated considering the results of both, brush cytologies and forceps biopsies. Since there were no false-positive diagnoses, specificity and positive predictive values reached $100 \%$.

Performance of stricture dilatation had a significant influence on the duration of ERC (mean duration of ERC without dilatation: $57.17 \pm 24.92 \mathrm{~min}$, mean duration of ERC with dilatation: $69.18 \pm 37.37 \mathrm{~min}, \mathrm{p}=0.04$ ).

Other potential confounding factors. Stricture localization, extent and length. Stricture localization and the respective diagnostic approach are summarized in Table 3. 92.02\% (173/188) of the strictures were high-grade with a narrowing $>90 \%$, whereas $7.98 \%(15 / 188)$ of the strictures showed a minor narrowing $<90 \%$. The mean stricture length was $22.45 \pm 10.79 \mathrm{~mm}$. Stricture length did not correlate with presence of malignancy (point-biserial correlation coefficient $r_{p b}=0.03$ ). Neither stricture localization nor extent nor length (strictures shorter vs. longer than mean length) significantly influenced the sensitivity of BC or FB. 


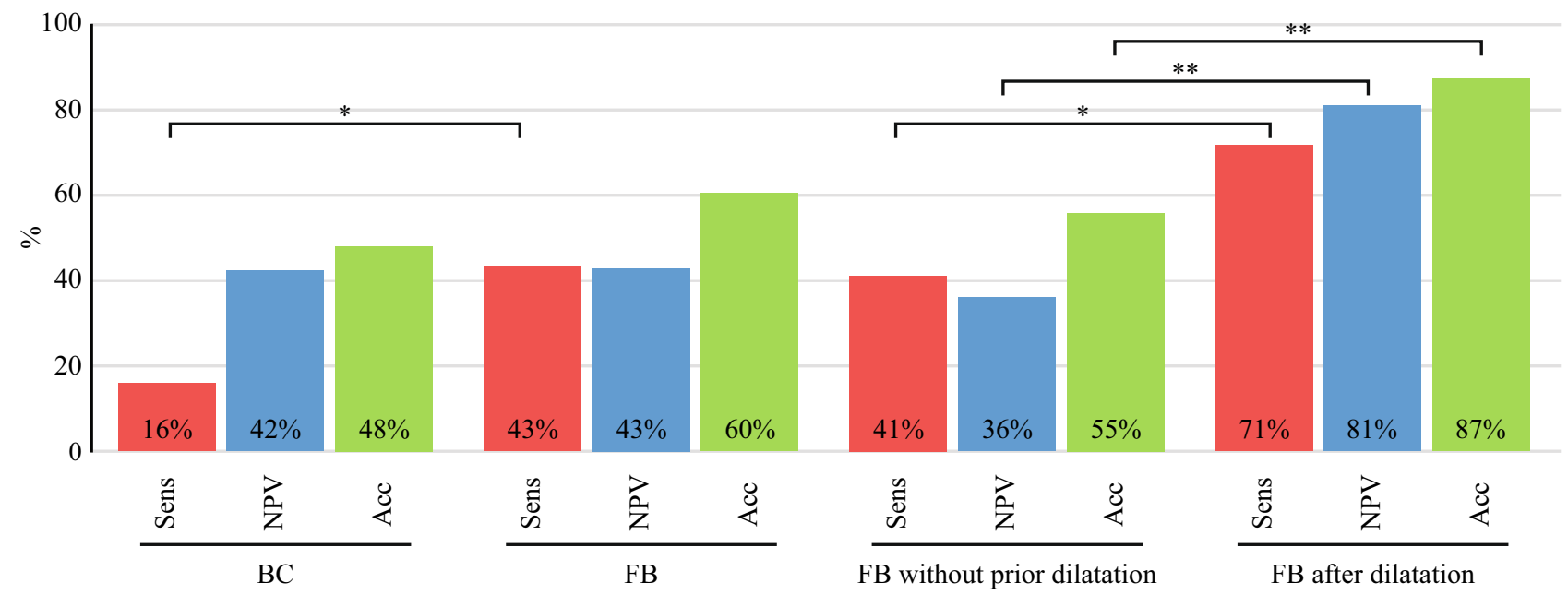

Figure 4. Diagnostic performance of $\mathrm{BC}$ and FB. The bar chart opposes the sensitivities (Sens), the negative predictive values (NPV) and the accuracies (Acc) of the respective modalities in percentages (rounded to the nearest whole number). Appendant specificities and positive predictive values were not depicted in this chart, as they were $100 \%$ consistently. $F B$ forceps biopsy, $B C$ brush cytology, ${ }^{\star} \mathrm{p}<0.05,{ }^{* *} \mathrm{p}<0.01$.

\begin{tabular}{|c|c|c|c|c|}
\hline & \multicolumn{4}{|c|}{ Stricture localization } \\
\hline & Distal $(n=87)(\%)$ & Perihilar $(n=84)(\%)$ & Intrahepatic ducts $(\mathrm{n}=25)(\%)$ & Others $^{\dagger}(n=22)(\%)$ \\
\hline $\mathrm{BC}(\mathrm{n}=83)$ & $27.59(24 / 87)$ & $39.29(33 / 84)$ & $52(13 / 25)$ & $59.09(13 / 22)$ \\
\hline $\mathrm{FB}^{\ddagger}(\mathrm{n}=188)$ & $94.25(82 / 87)$ & $84.52(71 / 84)$ & $72(18 / 25)$ & $77.27(17 / 22)$ \\
\hline \multicolumn{5}{|c|}{ Utilized forceps type for FB depending on stricture localization ${ }^{*}$} \\
\hline Large diameter forceps $(\mathrm{n}=111)$ & $88.89(48 / 54)$ & $79.63(43 / 54)$ & $68.75(11 / 16)$ & $60(9 / 15)$ \\
\hline Small diameter forceps $(n=35)$ & $14.81(8 / 54)$ & $27.78(15 / 54)$ & $37.50(6 / 16)$ & $40(6 / 15)$ \\
\hline $\begin{array}{l}\text { SpyBite Biopsy Forceps (Boston } \\
\text { Scientific, } n=8 \text { ) }\end{array}$ & $3.70(2 / 54)$ & $5.56(3 / 54)$ & $12.50(2 / 16)$ & $6.67(1 / 15)$ \\
\hline
\end{tabular}

Table 3. Frequencies of the applied modalities for transpapillary tissue sampling depending on stricture localization. $B C$ brush cytology, $F B$ forceps biopsy. ${ }^{\dagger}$ Including multilocular strictures and such involving the whole common bile duct. ${ }^{\ddagger}$ Forceps type could be determined in 139/188 cases retrospectively. Multiple forceps types were used in 15/139 cases.

Forceps types. Large diameter forceps and small diameter forceps were used in $79.86 \%(111 / 139)$ and $25.18 \%$ (35/139) of the cases (when FB was conducted and forceps type known), respectively (Table 3). Sufficient sample material for pathological examination was acquired in $99.10 \%(110 / 111)$ and $100 \%(35 / 35)$ of the cases using large and small diameter forceps, respectively. Their diagnostic performance did not differ significantly.

Effect of prior EST and prior biliary stenting. 119 of 211 (56.40\%) patients underwent ERC with endoscopic sphincterotomy (EST) prior to the evaluation period or prior to meeting the inclusion requirements for this study. Prior biliary stenting was done in 71 of 218 (32.57\%) patients. Neither prior EST nor prior stenting altered sensitivity of BC or FB significantly.

Implications of pre-interventional CA 19-9 levels on the diagnostic performance of brush cytology and forceps biopsy. Pre-interventional CA 19-9 levels of patients with malignant disease $(4218.72 \pm 11,828.27 \mathrm{U} / \mathrm{ml})$ were significantly higher than in patients with benign condition $(92.95 \pm 365.98 \mathrm{U} / \mathrm{ml}$, $\mathrm{p}<0.05$; see Table 1). ROC analysis provided a suitable cut-off value of $80 \mathrm{U} / \mathrm{ml}$ with a remarkable capacity to predict benign and malignant condition (AUC: $0.85 \pm 0.04$, sensitivity: $73.91 \%$, specificity: $85.71 \%$, PPV: 93.15\%, NPV 55.56\%, accuracy 77.17\%). When the cut-off value was exceeded, NPV and accuracy of BC and FB decreased significantly (BC: sensitivity: $0 \%$ vs. $14.29 \%$, p > 0.05, NPV: $65 \%$ vs. $5.26 \%$, p < 0.01 , accuracy: $65 \%$ vs. $18.18 \%$, p < 0.01 ; FB: sensitivity: $38.10 \%$ vs. $44.07 \%$, p > 0.05, NPV: $64.86 \%$ vs. $13.16 \%$, p < 0.01 , accuracy: $71.11 \%$ vs. $48.44 \%, \mathrm{p}<0.05$; each comparison for CA $19-9$ lower vs. higher than $80 \mathrm{U} / \mathrm{ml}$ ).

Adverse events. $\quad$ ERC-induced pancreatitis or cholangitis was observed after 14 of 242 (5.79\%) and 3 of 244 (1.23\%) ERCs, respectively (Table 4). ERC-induced perforation of the bile duct, severe post-interventional situations (hemorrhage requiring transfusions; requirement of intensive care or surgery) or death did not occur in 


\begin{tabular}{|l|l|l|}
\hline \multicolumn{2}{|l|}{} & \multicolumn{2}{|l|}{ Incidence of ERC-induced } \\
\cline { 2 - 3 } & Pancreatitis (\%) & Cholangitis (\%) \\
\hline Total $\left(\mathrm{n}=247^{\dagger}\right)$ & 5.79 & 1.23 \\
\hline Depending on stricture dignity & 6.94 & 1.37 \\
\hline Malignant stricture $(\mathrm{n}=149)$ & 4.35 & 0 \\
\hline Benign stricture $(\mathrm{n}=69)$ & 9.68 & 0 \\
\hline Depending on applied modality & 4.59 & 1.80 \\
\hline BC solely $\left(\mathrm{n}=31^{\dagger}\right)$ & 2.99 & 2.90 \\
\hline FB solely $\left(\mathrm{n}=113^{\dagger}\right)$ & 11.11 & 0 \\
\hline Large diameter FB only $\left(\mathrm{n}=69^{\dagger}\right)$ & \multicolumn{2}{|l}{} \\
\hline Small diameter FB only $\left(\mathrm{n}=9^{\dagger}\right)$ & 5.76 & 1.55 \\
\hline Depending on stricture dilatation & 5.88 & 0 \\
\hline No dilatation $\left(\mathrm{n}=196^{\dagger}\right)$ & \multicolumn{2}{|l}{} \\
\hline With dilatation $\left(\mathrm{n}=51^{\dagger}\right)$ & & \\
\hline
\end{tabular}

Table 4. Incidence of adverse events depending on stricture dignity, applied modality and stricture dilatation. $E R C$ endoscopic retrograde cholangiography, $B C$ brush cytology, $F B$ forceps biopsy. ${ }^{\dagger}$ Strictures with unconfirmed dignity included.

any case. Stricture dignity, applied modality or stricture dilatation showed no correlation with the incidence of adverse events.

\section{Discussion}

In suspected malignant biliary strictures tissue acquisition is usually required, since in most cases imaging alone with CT, MRI or EUS does not lead to a definitive diagnosis, which is albeit essential for initiating an appropriate therapy ${ }^{8,9}$. Furthermore, obtaining a histology in case of biliary malignancy is becoming increasingly important to assess the presence of predictive markers in order to offer a personalized chemotherapy, such as IDH1 with recently presented positive phase III data in the 2 nd line therapy ${ }^{10,11}$.

Endoscopic or percutaneous fine needle biopsy is a viable option in case of a visible mass or bile duct wall thickening ${ }^{12-14}$; but most patients with IBiS suffer from cholestasis or even septic cholangitis and require biliary drainage preferably through ERC. In this situation according to current recommendations ${ }^{4,6}$ transpapillary BC or FB is the primary method to obtain tissue for a cytological or histological diagnosis. While the specificity of both methods is excellent, sensitivity and NPV are limited.

In the present study we analyzed retrospectively a cohort of 218 patients, who underwent ERC with BC and/ or FB due to a suspected malignant biliary stricture between January 2010 and April 2018, in order to identify parameters that may impact the sensitivity and NPV of tissue sampling. In a metaanalysis from 2015 (including 9 studies and 730 patients) Navaneethan et al. ${ }^{15}$ reported comparable pooled sensitivities of BC and FB in diagnosing malignant biliary strictures of $45 \%$ and $48.1 \%$. Our data confirm this observation in a large and representative real-life cohort with a higher sensitivity of FB (43.4\%) compared to BC (15.9\%) while the specificity was $100 \%$ for both methods. Not surprisingly, we also found higher sensitivities for CCA (BC: $23.08 \%$, FB: $51.90 \%$ ) than for PCA (BC: $10 \%$, FB: 32.35\%). This difference might be explained by the fact that for technical reasons the brush detaches the cell material rather superficially, while the forceps penetrates deeper into the targeted tissue. In many cases with submucosal tumor growth or marked desmoplastic fibrosis and especially in case of an extraluminal tumor like PCA, the cell material obtained by BC might be suboptimal. It should be noted, however, that the significant difference between $\mathrm{BC}$ and $\mathrm{FB}$ in our cohort was primarily due to the comparatively poor sensitivity of $\mathrm{BC}$, while $\mathrm{FB}$ had a sensitivity that was comparable with previously reported ones ${ }^{15}$. This might be partly explained by the fact that we did not routinely use advanced cytologic techniques, such as digital image analysis and fluorescence in situ hybridization, which improve the sensitivity of BC for diagnosing malignancy in IBiS ${ }^{16-18}$. Moreover, we obtained adequate material for cytological examination in only $85.54 \%$ with brushing, which might be due to the relative high percentage of very fibrotic PSC related strictures. Other centers, however, reported excellent results of ERC with BC in screening for PSC related biliary malignancy ${ }^{19}$. Furthermore, we have used BC much less frequently, so that a certain selection bias cannot be excluded. It is therefore questionable whether our results should generally lead to the conclusion that $\mathrm{FB}$ is superior to BC. Rather, local cytopathological expertise as well as anatomical and technical conditions should also be considered. In order to optimize the cellular yield and thereby increase its sensitivity, some technical improvements of the brush have also been presented in the meantime ${ }^{20,21}$.

Given the limited sensitivity of $\mathrm{BC}$ and $\mathrm{FB}$ and in order to avoid false negative results, it is recommended either to use an alternative method like EUS-FNB for tissue sampling ${ }^{5,6}$ or to repeat the ERC with FB/BC (supplemented if necessary with advanced imaging techniques like cholangioscopy or intraductal ultrasound). Our findings support the latter recommendation: Taking into account an inevitable patient drop-out due to the clinical course of malignant disease, confirmation of stricture dignity through other modalities or a loss of follow-up, repetition of tissue sampling via ERC led to satisfying NPVs (with the $4^{\text {th }}$ ERC yielding an NPV of $88.89 \%$, see Fig. 3). As we could show, pre-interventional CA 19-9 levels proved to be a valuable tool in order to assess the reliability of negative cytological/histological findings of BC or FB. The NPV of BC and FB decreased significantly 
when a cut-off value of $80 \mathrm{U} / \mathrm{ml}$ was exceeded. Negative cytological/histological findings of BC or FB despite exceedance of the CA 19-9 cut-off value of $80 \mathrm{U} / \mathrm{ml}$ should encourage to repeat tissue sampling.

When we retrospectively analyzed a potential impact of other confounding co-variates, we found no significant impact of prior EST or prior biliary stenting on the sensitivity of BC or FB. In contrast to others ${ }^{22-24}$ we could also not confirm a significant impact of stricture length, stricture localization or stricture extent on the sensitivity of transpapillary tissue sampling. Also, sensitivity of FB did not differ between a large or small diameter forceps and the sample yield was excellent with both types. Nevertheless, according to previous publications modification of forceps type ${ }^{25,26}$ might even improve sensitivity or make it easier to insert the forceps into the bile duct and to reach the stricture. The most relevant finding in our study is probably the significantly better sensitivity and NPV of FB following prior balloon dilation (which was primarily indicated in order to secure bile flow and to allow the implantation of a biliary stent). In our study, we performed FB after balloon dilatation in 31 cases and without prior dilatation in 158 cases (Fig. 4). This somewhat more aggressive approach increased the duration of ERC $(57.17 \pm 24.92 \mathrm{~min}$ vs. $69.18 \pm 37.37 \mathrm{~min}, \mathrm{p}=0.04)$ but was not associated with an increased complication rate such as perforation, bleeding or pancreatitis (Table 4$)$. However, the sensitivity [71.43\% vs. $40.52 \%(\mathrm{p}=0.03)]$, the NPV $[80.95 \%$ vs. $35.51 \%(\mathrm{p}<0.01)]$ and the accuracy $[87.10 \%$ vs. $55.19 \%(\mathrm{p}<0.01)]$ were significantly higher for FB with prior balloon dilatation compared to FB without prior dilatation (Fig. 4). A potential selection bias cannot be excluded since balloon dilatation of the stricture was performed in only $49 / 218$ cases $(22.48 \%)$ and more likely in narrow strictures with relevant impairment of the bile flow. However as noted above, stricture extent did not have a significant influence on the diagnostic performance of FB in our study. Cholangiocarcinoma, the most common cause of IBiS in our study, often shows a periductal-infiltrating growth pattern with a dense desmoplastic fibrous stroma ${ }^{27}$. We speculate that balloon dilation tears the superficial desmoplastic and fibrous tissue so that the forceps can reach better and more effectively the submucosal tumor parts. Two previous studies analyzed the impact of stricture dilatation on the diagnostic yield: The first study ${ }^{28}$ on 46 patients reported that a combination of stricture dilation, endoscopic needle aspiration, and biliary brushing significantly improved the diagnostic yield for IBiS; the second study ${ }^{29}$ (139 patients) could not confirm a better sensitivity of BC after dilatation. In the present study we did not perform a balloon dilatation prior BC, because we expected that it is more difficult for the bristles of the brush to scrape cellular material from a stenosis that is then no longer narrowed. Therefore, in contrast to both previous studies, our study is the first which analyzes the effect of prior balloon dilatation on the sensitivity of forceps biopsy.

In conclusion, FB turned out to be the more effective method for transpapillary tissue sampling in our center. Balloon dilatation of the stenosis before FB provided a significant diagnostic benefit and was not associated with more complications. When both, tissue sampling and stricture dilatation, are indicated, we therefore recommend to dilate first and to take the FB afterwards. In order to validate our findings, we would advocate a prospective case control study. For this study design, the recruitment of patients will certainly take several years and could be accelerated by a contribution of multiple centers to the study.

\section{Data availability}

The datasets used and/or analyzed during the current study are available from the corresponding author on reasonable request.

Received: 16 August 2020; Accepted: 29 September 2020

Published online: 15 October 2020

\section{References}

1. Novikov, A., Kowalski, T. E. \& Loren, D. E. Practical management of indeterminate biliary strictures. Gastrointest. Endosc. Clin. N. Am. 29, 205-214. https://doi.org/10.1016/j.giec.2018.12.003 (2019).

2. Weismüller, T. J. et al. Patient age, sex, and inflammatory bowel disease phenotype associate with course of primary sclerosing cholangitis. Gastroenterology 152, 1975-1984.e8. https://doi.org/10.1053/j.gastro.2017.02.038 (2017).

3. Tischendorf, J. J. W., Hecker, H., Krüger, M., Manns, M. P. \& Meier, P. N. Characterization, outcome, and prognosis in 273 patients with primary sclerosing cholangitis: a single center study. Am. J. Gastroenterol. 102, 107-114. https://doi.org/10.111 1/j.1572-0241.2006.00872.x (2007).

4. Kempeneers, M. A. et al. International consensus guidelines for surgery and the timing of intervention in chronic pancreatitis. Pancreatology 20, 149-157. https://doi.org/10.1016/j.pan.2019.12.005 (2020).

5. Nakai, Y. et al. International consensus statements for endoscopic management of distal biliary stricture. J. Gastroenterol. Hepatol. 35, 967-979. https://doi.org/10.1111/jgh.14955 (2020).

6. Sun, B. et al. Review article: Asia-pacific consensus recommendations on endoscopic tissue acquisition for biliary strictures. Aliment Pharmacol. Ther. 48, 138-151. https://doi.org/10.1111/apt.14811 (2018).

7. Kulaksiz, H. et al. A novel method of forceps biopsy improves the diagnosis of proximal biliary malignancies. Dig. Dis. Sci. 56, 596-601. https://doi.org/10.1007/s10620-010-1535-4 (2011).

8. Heinzow, H. S. et al. Comparative analysis of ERCP, IDUS, EUS and CT in predicting malignant bile duct strictures. World J. Gastroenterol. 20, 10495-10503. https://doi.org/10.3748/wjg.v20.i30.10495 (2014)

9. Rösch, T. et al. A prospective comparison of the diagnostic accuracy of ERCP, MRCP, CT, and EUS in biliary strictures. Gastrointest. Endosc. 55, 870-876. https://doi.org/10.1067/mge.2002.124206 (2002).

10. Lowery, M. A. et al. Safety and activity of ivosidenib in patients with IDH1-mutant advanced cholangiocarcinoma: a phase 1 study. Lancet Gastroenterol. Hepatol. 4, 711-720. https://doi.org/10.1016/S2468-1253(19)30189-X (2019).

11. Abou-Alfa, G. K. et al. ClarIDHy: a global, phase III, randomized, double-blind study of ivosidenib (IVO) vs placebo in patients with advanced cholangiocarcinoma (CC) with an isocitrate dehydrogenase 1 (IDH1) mutation. Ann. Oncol. 30, v872-v873. https ://doi.org/10.1093/annonc/mdz394.027 (2019).

12. Garrow, D. et al. Endoscopic ultrasound: a meta-analysis of test performance in suspected biliary obstruction. Clin. Gastroenterol. Hepatol. 5, 616-623. https://doi.org/10.1016/j.cgh.2007.02.027 (2007).

13. Lee, J. H., Salem, R., Aslanian, H., Chacho, M. \& Topazian, M. Endoscopic ultrasound and fine-needle aspiration of unexplained bile duct strictures. Am. J. Gastroenterol. 99, 1069-1073. https://doi.org/10.1111/j.1572-0241.2004.30223.x (2004). 
14. Mohamadnejad, M. et al. Role of EUS for preoperative evaluation of cholangiocarcinoma: a large single-center experience. Gastrointest. Endosc. 73, 71-78. https://doi.org/10.1016/j.gie.2010.08.050 (2011).

15. Navaneethan, U. et al. Comparative effectiveness of biliary brush cytology and intraductal biopsy for detection of malignant biliary strictures: a systematic review and meta-analysis. Gastrointest. Endosc. 81, 168-176. https://doi.org/10.1016/j.gie.2014.09.017 (2015).

16. Barr Fritcher, E. G. et al. An optimized set of fluorescence in situ hybridization probes for detection of pancreatobiliary tract cancer in cytology brush samples. Gastroenterology 149, 1813-1824.e1. https://doi.org/10.1053/j.gastro.2015.08.046 (2015).

17. Levy, M. J. et al. Prospective evaluation of advanced molecular markers and imaging techniques in patients with indeterminate bile duct strictures. Am. J. Gastroenterol. 103, 1263-1273. https://doi.org/10.1111/j.1572-0241.2007.01776.x (2008).

18. Smoczynski, M. et al. Routine brush cytology and fluorescence in situ hybridization for assessment of pancreatobiliary strictures. Gastrointest. Endosc. 75, 65-73. https://doi.org/10.1016/j.gie.2011.08.040 (2012).

19. Boyd, S. et al. Screening primary sclerosing cholangitis and biliary dysplasia with endoscopic retrograde cholangiography and brush cytology: risk factors for biliary neoplasia. Endoscopy 48, 432-439. https://doi.org/10.1055/s-0041-110792 (2016).

20. Fogel, E. L. et al. Effectiveness of a new long cytology brush in the evaluation of malignant biliary obstruction: a prospective study. Gastrointest. Endosc. 63, 71-77. https://doi.org/10.1016/j.gie.2005.08.039 (2006).

21. Shieh, F. K. et al. Improved endoscopic retrograde cholangiopancreatography brush increases diagnostic yield of malignant biliary strictures. World J. Gastrointest. Endosc. 6, 312-317. https://doi.org/10.4253/wjge.v6.i7.312 (2014).

22. Naitoh, I. et al. Predictive factors for positive diagnosis of malignant biliary strictures by transpapillary brush cytology and forceps biopsy. J. Dig. Dis. 17, 44-51. https://doi.org/10.1111/1751-2980.12311 (2016).

23. Nishikawa, T. et al. Factors affecting the accuracy of endoscopic transpapillary sampling methods for bile duct cancer. Dig. Endosc. 26, 276-281. https://doi.org/10.1111/den.12140 (2014).

24. Park, J. G. et al. Percutaneous transluminal forceps biopsy in patients suspected of having malignant biliary obstruction: factors influencing the outcomes of 271 patients. Eur. Radiol. 27, 4291-4297. https://doi.org/10.1007/s00330-017-4796-x (2017).

25. Tamada, K. et al. Ropeway-type bile duct biopsy forceps with a side slit for a guidewire. Gastrointest. Endosc. 53, 89-92. https:// doi.org/10.1067/mge.2001.112094 (2001).

26. Yamamoto, K. et al. Evaluation of novel slim biopsy forceps for diagnosis of biliary strictures: Single-institutional study of consecutive 360 cases (with video). World J. Gastroenterol. 23, 6429-6436. https://doi.org/10.3748/wjg.v23.i35.6429 (2017).

27. Rizvi, S. \& Gores, G. J. Current diagnostic and management options in perihilar cholangiocarcinoma. Digestion 89, 216-224. https ://doi.org/10.1159/000360791 (2014)

28. Farrell, R. J. et al. The combination of stricture dilation, endoscopic needle aspiration, and biliary brushings significantly improves diagnostic yield from malignant bile duct strictures. Gastrointest. Endosc. 54, 587-594. https://doi.org/10.1067/mge.2001.118715 (2001).

29. de Bellis, M. et al. Influence of stricture dilation and repeat brushing on the cancer detection rate of brush cytology in the evaluation of malignant biliary obstruction. Gastrointest. Endosc.. 58, 176-182. https://doi.org/10.1067/mge.2003.345 (2003).

\section{Acknowledgements}

Interim results of this study were presented at the 73rd annual conference of the German Society of Gastroenterology (DGVS) in 2018 and the 49th congress of the German Society of Endoscopy (DGE-BV) in 2019. The present study is part of the MD thesis of D. Pörner.

\section{Author contributions}

D.P. and T.J.W. designed and initiated the study, enrolled patients, performed the statistical analysis, analyzed the results and wrote and edited the paper. D.J.K., D.H., A.H., R.M., R.H., J.N., C.P.S. and T.J.W. performed the endoscopic interventions. H.M., T.R.G. and S.M. performed the surgical interventions during the clinical follow-up. M.A.G. did the oncological follow-up and treatment. H.P.F. and M.T. performed the histopathological analyses. All authors approved the final draft of the manuscript.

\section{Funding}

Open Access funding enabled and organized by Projekt DEAL.

\section{Competing interests}

The authors declare no competing interests.

\section{Additional information \\ Correspondence and requests for materials should be addressed to T.J.W.}

Reprints and permissions information is available at www.nature.com/reprints.

Publisher's note Springer Nature remains neutral with regard to jurisdictional claims in published maps and institutional affiliations.

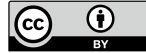

Open Access This article is licensed under a Creative Commons Attribution 4.0 International License, which permits use, sharing, adaptation, distribution and reproduction in any medium or format, as long as you give appropriate credit to the original author(s) and the source, provide a link to the Creative Commons licence, and indicate if changes were made. The images or other third party material in this article are included in the article's Creative Commons licence, unless indicated otherwise in a credit line to the material. If material is not included in the article's Creative Commons licence and your intended use is not permitted by statutory regulation or exceeds the permitted use, you will need to obtain permission directly from the copyright holder. To view a copy of this licence, visit http://creativecommons.org/licenses/by/4.0/.

(C) The Author(s) 2020 